\title{
The Zones of Proximal and Distal Development in Chinese Language studies with the use of wikis
}

\author{
Esyin Chew \\ Centre for Excellence in Learning and Teaching (CELT), University of South Wales, United Kingdom; \\ School of Information Technology, Monash University Sunway Campus, Malaysia.
}

\author{
Seong Lin Ding \\ Faculty of Languages and Linguistics, University of Malaya, Malaysia
}

\begin{abstract}
Educational practitioners in the higher education institutions of the UK have increasingly promoted the use of wikis. The technology enhanced learning experience of the UK was transferred to a local higher educational agency in Malaysia through a collaborative research project called WiLearn. By examining a student cohort enrolled in Chinese language studies, WiLearn explores the Zone of Proximal Development (ZPD) and the Zone of Distal Development (ZDD) with regard to the use of wikis in peer reviewed group coursework. With the goal of informing higher education researchers and practitioners, the problematic use of wikis was discussed according to the following three dimensions: (a) the process of group work; (b) social presence; and (c) the outcome of group work. The findings reveal that more critical reflection is evident when retrieving peers' comments through WiLearn, but less critical discourse is evident among the participants. The difference between the ZPD and the ZDD lies not in the functional use of wikis but in the degree of openness and social presence of the students from Chinese language studies. A pedagogical change in critical peer review and discourse regarding the use of wikis is suggested. This paper concludes with constructive and disruptive lessons that were learned through a series of insights that provide a greater understanding of the challenges and opportunities with regard to learning and teaching with wikis for Chinese language studies.
\end{abstract}

\section{Background}

Web 2.0 technologies are growing vigourously and are increasingly being used in higher education. One of the most powerful web 2.0 technologies is the wiki. The wiki represents an online platform that provides conversational knowledge development and sharing (Wagner, 2004). Wikis effectively support community-oriented authoring and editing to create easy interactive learning environments for the development of information (Richardson, 2006). Users of wikis can rapidly develop and edit the wikis' content, and the wikis' history feature is helpful for users to track the development of the content and the timing of the editing (Shiha, Tsenga, \& Yangc, 2008; Lai \& Ng, 2011). With these flexible features, wikis provide collective authoring and contribute to the process of peer reviewing. Xiao and Lucking (2008) suggest that wikis insert value to peer assessment by providing an online collaborative learning environment that facilitates peer reviewing. Similarly, Laughton (2011) articulates that wikis' features enhance collaboration among learners and further facilitate the opportunity to learn from peers. Wikis are more than just a tool for online group work, given that they provide a platform for community learning and development. Guth (2007) asserts that wikis facilitate learners' development of the art of constructive criticism, which decreases their dependency on educators' guidance and feedback. Similarly, Leading (2010) suggests that the educators' responsibility to provide feedback has been shifted to the learners due to the responsible use of wiki technology. Given the various benefits of wikis, western educational practitioners and researchers have increasingly promoted the use of wikis in learning and teaching in higher education (Bold, 2006; Richardson, 2006; Lamb \& Johnson, 2007; Ajjan \& Hartshorne, 2008; Robertson, 2008; Haley, Collins, \& Coe, 2008; JISC, 2011). It is no longer just an innovative idea, as it has become a promising instructional approach for collaborative work. Based on a review of previous literature, Zokor (2009) asserts that educators are able to monitor students' group or individual progress with the use of wikis. By monitoring students' participation, educators can more readily assess the students' work. In addition, there is the possibility that students can fundamentally change their own knowledge construction and develop a deeper understanding of concepts through transformative dialogues. Zokor claims that this construction of new knowledge makes wikis "transformative software" (pp. 649-650). 
In contrast, Conole (2010) asserts that the uptake of web 2.0 technologies in learning and teaching has being marginal thus far. Hwang and Brummans (2011) argue that "comparatively few studies have looked at the actual experiences of students who are engaged in building a wiki community for a particular course" (p. 39). Limited research has focused on the learning effects of wikis or on the failed experience of wikis with regard to learning. In a study from the UK, Cole (2009) reports a failed experimental use of wikis to support learning in an undergraduate module and offers some insights into the pitfalls of embedding wikis in learning and teaching. Given the diversity in culture, epistemology and social practices between the east and west, more research that draws from Asian experiences is needed with regard to using promising technology, such as wikis $(\mathrm{Ng} \mathrm{\&} \mathrm{Lim,} \mathrm{2011).} \mathrm{To} \mathrm{date,} \mathrm{richer} \mathrm{pedagogical}$ insight regarding the use of wikis to enhance actual students' learning experiences (or not) in diverse contexts has not yet been provided.

The UK's higher educational institutions (HEIs) have a good track record regarding research in and practice with integrating technology to enhance learning and teaching (Laurillard, 2002; Salmon, 2002; Sharpe, Benfield, Roberts, \& Francis, 2006; Conole, Laat, Dillon, \& Darby, 2008). Gwella (2010) and JISC (2011) promote the innovative use of web 2.0 technologies across the HEIs and enable research communities to engage in national and global collaborations to overcome the challenges of delivering a technology enhanced learning (TEL) agenda. In Malaysia, "e-learning" is the term used in the place of "TEL". In line with the Malaysian Ministry of Higher Education's policy (Mohamed, 2011), HEIs are determined to increase the number of courses that use e-learning platforms. The oldest local HEI in the country, the University of Malaya (UM), has committed to the following two missions: (a) to design and develop learning innovations and improvements and (b) to improve the efficiency and effectiveness of instruction through the application of research and technology, such as the UM Spectrum (http://spectrum.um.edu.my) in Moodle (ADeC, 2011). Thus, there appears to be a synergy between the UK and Malaysia's HEIs in terms of the TEL agenda. To bring the TEL experience from the UK to Malaysia, we respond to this synergy by conducting a cross-country collaborative study, called WiLearn, to investigate the effects of wiki use on students in Chinese language studies in Malaysia. The Faculty of Languages and Linguistics at UM offers a Bachelor of Languages and Linguistics programme with a specialisation in Chinese language studies, which focuses on providing courses in linguistics enhancement in Chinese. Academic courses include the history and development of the Chinese language, the Chinese language for special purposes, translations, sociolinguistics and so on. Upon completion of the programme, graduates are equipped with a high degree of proficiency in Chinese language studies and have good interpersonal communication and research skills.

\section{Theoretical framework: is the wiki an effective tool for developing the Zone of Proximal Development?}

To embed wikis in learning and teaching practices, it is necessary to explore this innovative tool under the guidance of sound learning theories. There are many learning theories that have emerged in the last few decades and each has arguments that are diverse and complex. Constructivism is commonly associated with learning technology. Previous literature suggests that the wiki is an excellent tool for constructive learning and working collaboratively (Engstrom \& Jewett, 2005; Murugesan, 2007; West \& West, 2009). By providing an example of how technology supports large group interactions during socially constructive learning activities, Weaver, Keer, Schellens and Valcke (2011) assert that it is feasible to integrate intra-group peer assessment with the use of wikis in higher education. It is generally believed that Vygotsky's educational insights are best suited for social constructivism and TEL (Pear \& CroneTodd, 2002; Subramaniam, 2007; Jones, Blackey, Fitzgibbon, \& Chew, 2010; Nussbaum, Alvarez, McFarlane, Gomez, Claro, \& Radovic, 2009; Hull \& Saxon, 2009). Vygotsky (1978) suggests that learners' knowledge is developmentally constructed during social and cultural interactions; therefore, cognitive skills are mediated by words and language, which serve as psychological tools for facilitating and transforming mental activity in social-cultural relations (Cortazzi, 1999; Deborah \& Bodrova, 2001). Vygotsky perceives language as the substantive mediator that humans use to share social meanings with one another and to explain how learners advance developmentally from natural processes to higher mental processes (Jaramillo, 1996). The Zone of Proximal Development (ZPD) is Vygotsky's term for a simple educational principle, which is that individuals' performances are enhanced when they are aided by knowledgeable individuals when compared to working independently (Leong \& Bodrova, 2007; Vygotsky, 1978). Several types of peer-assistance are needed to develop new skills and concepts within the learners' ZPD (Deborah \& Bodrova, 2001). 
In the light of Vygotsky's insights (1978), web 2.0 technologies, which include wikis, could further enhance the language learning process given its socially constructive platform for collective group thoughts within the ZPD. To paraphrase, learning enhancement for wiki users may be developmentally constructed in social and/or cultural interactions on wikis that have history features. Thus, cognitive skills are mediated by the text/language that is registered on wikis for developing mental activity in socialcultural relations. Grounded in this hypothesis, we further explore whether wikis serve the purpose of ZPD, as suggested by Vygotsky in the context of language studies, and determine the polarity if wikis defeat the ZPD. In this research, we propose a new antonym of ZPD to describe this defeat, which is the Zone of Distal Development (ZDD). This outcome is a disruptive aspect of socially constructive educational activities that are available in wikis. To constructively insert originality into the subject matter, we propose a relationship between two opposite attributes of the ZPD (i.e., learning construction) and the ZDD (i.e., learning disruption).

Richardson (2006) articulates that there are increasing opportunities to use wikis in language studies. Recent studies confirm that the use of educational technology, including wikis, is effective for language learning, primarily in English as a second language studies (Mak \& Coniam, 2008; Connolly, Stansfield, \& Hainey, 2011; Kim, 2011; Joubert \& Wishart, 2012). Nevertheless, there is little evidence available in a current review of the literature showing the effective use of wikis in Chinese language studies. Zokor's (2009) studies suggest that wikis can be used to enhance effective collaboration in a constructivist approach for all types of language learning. Yet, given differences in educational practices, pedagogical insights grounded in the ZPD and/or the ZDD for Chinese language students using wikis should be explored. The Specialisation of Chinese provides Malaysian students with comprehensive training in Chinese language studies by equipping them with both the conceptual and practical skills needed for further critical communication and professional tasks involving the use of Chinese. Hence, the following two main research questions are put forward in the present study:

1. What are the effects of the ZPD and/or the ZDD with regard to the use of wikis in Chinese language studies in Malaysia?;

2. What is the polarity of the ZPD and the ZDD based on the findings from (1)?

\section{Research design and method}

The action inquiry method (Torbert, 2001) was adapted for use in this study. First, the territory of inquiry resides in the following hypothesis: "with the use of wikis, learners' learning enhancement may be developmentally constructed during social interactions on wikis, and, therefore, cognitive skills are mediated by the text/language that is registered on the wikis for developing mental activity in socialcultural relations", which is the ZPD as suggested by Vygotsky (1978) in the context of Chinese language studies. Polarity (the ZDD aspects) is evident if the use of the wikis fails to create the ZPD. Borrowing from both McConnell (2006) and Zokor's (2009) analyses of wiki groups' collaboration, we created a coding scheme with three dimensions to investigate the effects of the ZPD and to determine whether wikis serve the purposes of the ZPD and/or the ZDD, as follows:

1. The process of group work (PGW): monitor students' group or individual progress by monitoring their participation, including their ability to develop in-depth discussions, to ask questions and to contribute to the group work with the use of wikis;

2. The social presence (SP): empower students with more responsibility and increase the degree of “openness" among wiki group participants by assessing individuals' engagement activities, their sharing of information and the public visibility of their work on wikis that facilitate sharing and construction of information, resources and ideas; and

3. The outcome of group work (OGW): demonstrate the results of the wiki group participants' final output of group work and the ideas expressed on wikis, including whether the students' use of wikis fundamentally changed their own knowledge constructions and whether it helped develop a deeper understanding of the concepts through transformative dialogue.

Some elements of these dimensions may overlap, for instance, the effects of PGW and SP may contribute to OGW. 
Second, within the research strategy, WiLearn is a collaborative research project between a UK researcher (i.e., the Senior Lecturer in technology enhanced learning, teaching and assessment in the UK) and a local agency (i.e., the Senior Lecturer in the Department of Malaysian Languages and Applied Linguistics in Malaysia). The UK's experience with TEL was shared and transferred through this collaborative research project with a local educational agency in Malaysia. By examining student cohorts who are enrolled in Chinese language studies, the WiLearn's objectives are the following: (1) embed wikis as a manner of innovative collaborative group work with peer feedback; (2) explore the effects of the ZPD and/or of objective 1 of the PGW, SP and OGW; and (3) investigate the polarity of the ZPD and the ZDD with regard to the first use of wikis in Chinese language studies in Malaysia. Prior to the use of wikis, technical and pedagogical training sessions were provided by the UK researcher to the local agency that detailed enhanced learning practices for wikis.

The undergraduate second year module, Chinese for a Specific Purpose, from the Bachelor of Languages and Linguistics programme (with a specialisation in Chinese language studies) was selected. Fifteen Malaysian Chinese students were enrolled in this module. These students were all Chinese native speakers who were aiming to advance their Chinese language skills for critical and journalistic writing. According to Vygotsky (1978), language is the important mediator that students use to facilitate and transform mental activity in social relations for the ZPD. The development of language advancement for selected research participants may be developmentally constructed during social interactions; therefore, cognitive skills are mediated by the language that is registered on wikis to develop more critical mental activity in journalistic writing. The inclusion of only Chinese native speakers as research participants removes the obstacle of using a "foreign language" in collaborative work, which may minimise the ZPD. In addition, this student cohort was chosen because they have no prior experience with using wikis during group work. Most of them have been participating in face-to-face meetings with books, journals and newspapers for critical group discussions. We believe that this small sample could provide fresh insight and represent the Chinese language students in Malaysia who generally have very limited prior experience with TEL. It is interesting to explore these students' ZPD and/or ZDD during the use of wikis, as there is a long history of relying on traditional "white board and pen" learning and teaching experiences during Chinese language studies in Malaysia. We did not include students with prior technological experience (such as students from the Faculty of Computer Science) who had no linguistic expertise in the critical group discussions and peer collaboration experience. It is worth noting that this was the students' first experience with the use of wikis for peer review and feedback in Chinese language studies in Malaysia.

Third, to perform the research, the local agency created wikis on UM Spectrum and students were required to complete group coursework using these wikis. The 15 students were subdivided into four groups to develop critical discussions using the wikis. There were four themes for the collaborative group work, as follows: (a) The 2010 Nobel Peace Prize awarded to Liu Xiaobo; (b) The situation occurring on the South Korean peninsula; (c) The survival of the Chilean miners (in contrast to mine accidents in China); and (d) Wikileaks. The learning objectives for this group work were to:

1. Use the diverse scope of themes to promote critical thinking and discussion by reflecting on Chinese politics, economics and culture, in contrast to global news; and

2. To enhance the traditional instructional method (i.e., face-to-face discourse without peer review) by using wikis when constructing critical information for sharing and peer review.

To assess the PGW, SP and OGW, learning process within the ZPD and ZDD were observed and analysed during and following the WiLearn research project. Qualitative data were collected using a semi-structured questionnaire, and all 15 students completed this questionnaire. The following are some examples of questions that were asked:

- Do you think that the wiki is helpful and/or enhances your learning experience, and, if so, how (PGW, SP, OGW)?

- What are your top negative experiences with regard to the wiki (PGW, SP, OGW)?

- Probe 1: Have you encountered any difficulties or 'disruptions' when using the wiki?

- Probe 2: Are there any technological limitations?

- Probe 3: Are there any pedagogical limitations?

- Do you think that the wiki enhanced your understanding of the knowledge/assessment requirements (when compared to the learning objectives)? 
- Probe: Do you think that you were more critical of your own contributions after having read others' comments about your contributions?

- Do you think that you were more critical of your own group work after using the wiki to provide comments to others in the same group? Please explain.

Four students were interviewed to capture their in-depth experiences based on the diversity of the effects regarding the ZPD and the ZDD. The three dimensions of the coding scheme, which were the PGW, SP and OGW, were employed to analyse the students' perceptions. Subthemes were extracted from the students' responses to inform the polarity of the ZPD and the ZDD. To ensure the reliability and validity of the data, we addressed several general questions repeatedly in the process of data collection and analysis, including the following: Are the findings replicated?; Are the answers consistent across different research participants?; Are all of the responses real and valid?; , and do they have integrity after cross checking with each other and with the observations during the interviews? In addition, we incorporated the following considerations and actions to improve validity and reliability during the data collection process and the site visits:

- Before data collection: Design issues that were identified by Denscombe (2002), including double-barrelled questions, similar questions asked in different fashions, ambiguous wording and technological or social science jargon, were prevented. A pilot test was conducted to refine and translate the interview questions by the UK researcher and the local agency.

- After data collection: Denscombe (2002) and Cohen, Manion \& Morrison (2001) stress that reliability issues, such as interviewer bias and misperception of responses, are major problems in qualitative research. Therefore, this study was designed such that: (a) the UK researcher doublecoded and analysed the data to prevent any bias of the local agency. However, based on Vaus (2001) and Taylor and Bogdan's (1998) views, it is almost impossible not to interpret quotes based on the researchers' selection of what they see as relevant and important. Therefore, validity and reliability were improved using (b) a triangulation method (Flick, 1998; Denscombe, 2002), in which a comparison of the observations, an analysis of the history of the wiki sites and individuals' engagement, activities and interview findings were used to explain the strengths and weaknesses of the current research. For example, over half of the participants indicated in the questionnaires that they wanted to use wikis during future group work. Employing in-class discussion and reflection, the local agency observed that most participants did not wish to use the wikis in future collaborative work. The positive views expressed on the questionnaires may have been influenced by the culture of Malaysian Chinese students to be "courteous" and appreciate the researchers and the local agency for their efforts to conduct the WiLearn research project. These particular data were, therefore, removed from coding and analysis.

\section{Results}

\section{General discussion - efficient retrieval of information from diverse resources}

Most of the participants stated that learning enhancement occurred with the use of wikis, regardless of whether it was major or minor enhancement. They generally perceived the wikis as a "one-stop-shop" for quick information retrieval, as the context and background of a topic were easy to understand. Yet, respondents also generally lacked confidence in the quality of the information found on the wikis due to issues with the reliability and validity of the sources. Some students asserted that the resources cited on the wiki were not suitable for use as scholarly references. As suggested by Waters (2007) and Soylu (2009), the use of wikis for research and coursework should be limited. In contrast, Anthony, Smith and Williamson (2005) and Wilkinson and Huberman (2007) argued that wiki resources are valid and reliable given the scholarly evidence. More students in the present study stated that the wikis offered objective information in a multi-faceted outlet that was outside of the traditional classroom. Consider the following opinion:

I could learn knowledge outside of the classroom through the wikis... and possibly a lot of undisclosed information! (Respondent 6 [R6]) 
Table 1

Supporting quotes for the themes extracted from the participants' responses

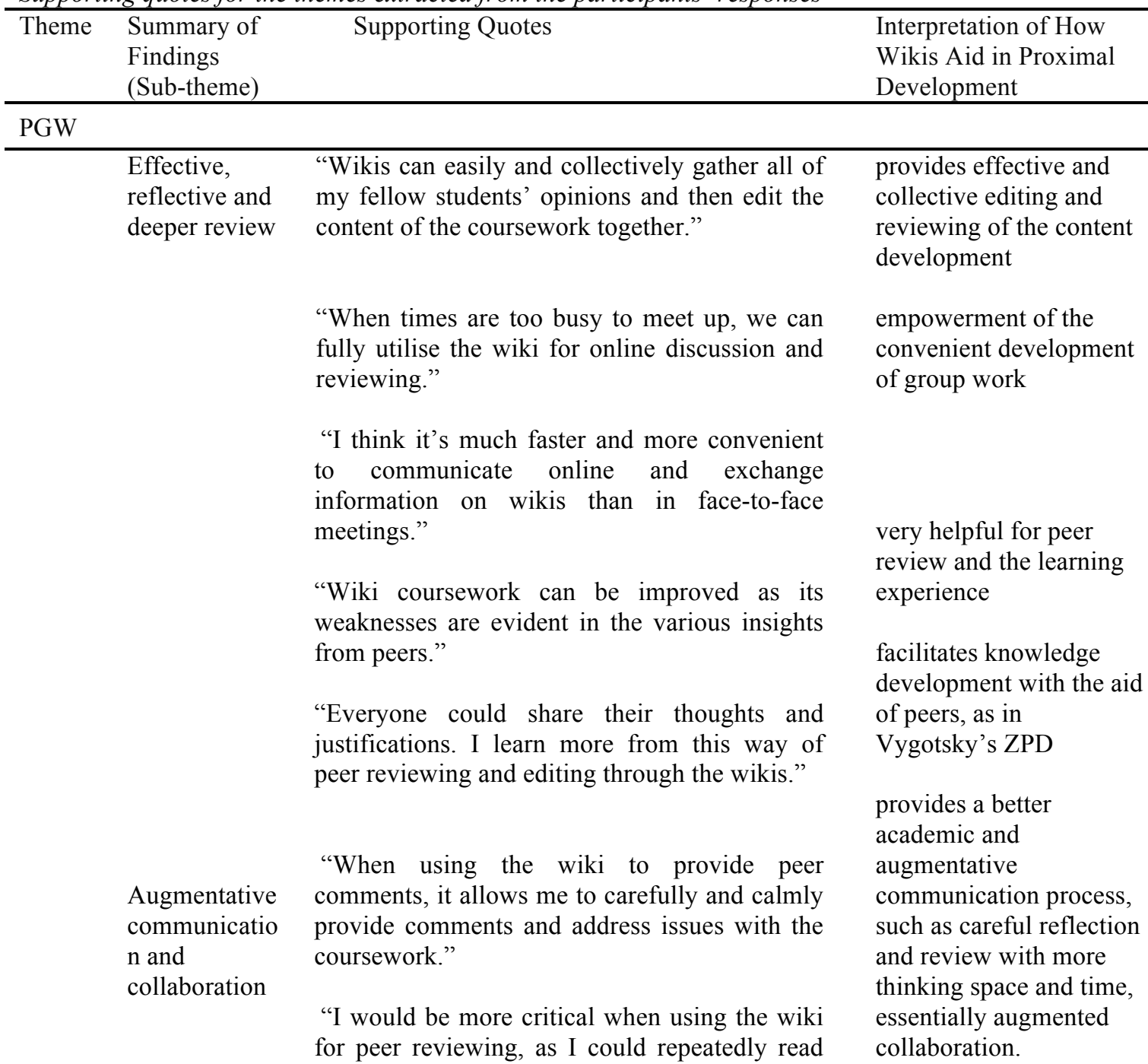
the collaborative work over and over again. Therefore, it's easier and more deliberate to find things that could be improved."

"I could 'listen' to others more clearly through the wiki...it is a convenient way to communicate."

"Without the use of the wiki, we could only provide superficial comments and discussion with simpler and perfunctory editing. Yet, there is the possibility that your peers may not adopt or accept your views [in face-to-face]."

SP

\begin{abstract}
Inclusive
learning
\end{abstract}

\author{
"Yes, I would like to use the wiki because I can \\ receive different opinions, and more useful, \\ concise and straight-forward comments than \\ intuitive feedback from face-to-face."
}

"This is a fair and unbiased method for open discussion and peer assessment"

\author{
facilitates inclusive \\ learning with an open \\ discussion space that is \\ without bias.
}


Collective information that students learn from wikis, which differs from the authoritative facts presented in the classroom, encourages higher levels of knowledge construction and academic debates. Legitimate sources for academic publications plus rapidly edited and open resources are powerful combined instruments for learning. The results reveal that, in general, research participants have a positive attitude towards the use of wikis for general purposes. They prefer to "access" and "retrieve" information from wikis rather than use wikis as a collaborative tool, given that most of them did not want to use the wikis in future collaborative work. This instrumental finding should be further analysed and discussed in light of the ZPD and the ZDD with regard to the dimensions of PGW and SP. The discussion of PGW and SP will be summarised to contribute to the OGW.

\section{Zone of Proximal Development (ZPD)}

In addition to an instrumental discussion, there are promising ZPD effects for WiLearn. Table 1 presents the opportunities to develop the ZPD, which were justified by the students' responses.

Students found the wiki to be very helpful for peer review and the learning experience. This finding supports Shiha et al. (2008), Zokor (2009) and Laughton's (2011) claims that wikis provide effective and collective editing and reviewing for content development. In addition to supporting the convenient development of group work, WiLearn also creates a certain level of Vygotsky's ZPD to facilitate knowledge development with the aid of peers. Compared with traditional face-to-face discussion, the present study shows that wikis can provide a better academic and augmentative process, which includes careful reflection and review with more thinking space and time, essentially augmented collaboration. Careful, calm and repeated peer review can be realised with the use of wikis. Moreover, a helpful feature of WiLearn for educators is the history page, which displays the differences between old and new versions of the wiki. This feature makes effectively monitoring the progress of individuals and groups possible. Figure 1 shows an example of a history page.

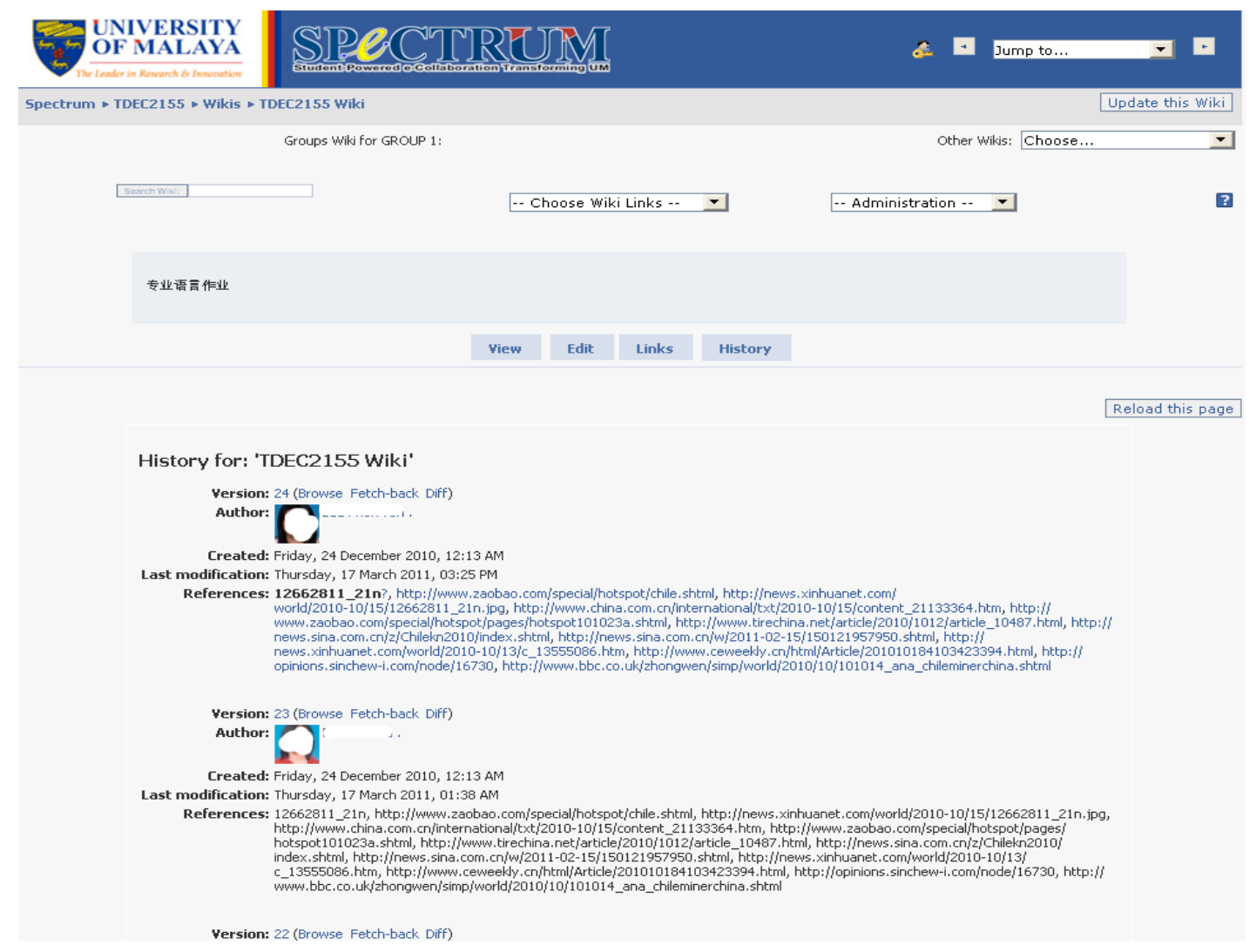

Figure 1. History page on WiLearn 


\section{Zone of Distal Development (ZDD)}

Progress of group work (PGW)

During the PGW, students collaboratively and critically developed the contents of the wiki and provided collective editing through the process of peer review. As previously discussed, many educational theorists and practitioners agree that learning is enhanced within a learning community and a socially constructive environment. One concern may be raised here, which is whether the peer-assisted learning process was enhanced by the wiki. If not, then face-to-face discussion for this type of peer review exercise is sufficient without the use of the wiki. This challenge was discussed in the following responses:

The wiki is useless for providing peer feedback. Group discussions during free time can achieve the same purposes!

It makes no difference at all if we did the face-to-face discussion in private.

I think that, rather than using the wiki, it is much more effective and time-saving to have face-to-face discussions, especially when it comes to debates and addressing issues and opinions for coursework.

In contrast to the benefit of convenient communication, a number of students thought that WiLearn sometimes added no value to the face-to-face discourse. Additionally, problems, such as comments that were less critical and overly simplistic, occurred during the peer review exercise. Some students may have participated in the peer assessment because it was an "official requirement" for assessment. One student asserted that "it would be lacking of real critical feedback if there is an enforcement applied to be critical". An obvious example of this involves one student who only logged into the wiki on one evening throughout the duration of the course. He simply edited peers' content with following justification:

[R15]: (14 March 8:12pm) The paragraph is edited due to repeated content and the sentence does not read smoothly.

[R15]: (14 March 8:17pm) The paragraph is edited due to repeated content and the sentence does not read smoothly.

[R15]: (14 March 8:35pm) The paragraph is edited due to repeated content.

[R15]: (14 March 8:55pm) The paragraph is edited due to repeated content.

This is a poor pedagogical practice for peer review and defeats the purposes of WiLearn. This challenge raises the following issues:

- How does one encourage students to fully utilise the wiki for learning in a critical and effective manner?;

- What distinct face-to-face discourse and collaborative activities work on wikis?

Some research participants prefer face-to-face discussion when compared to using the wiki. They are used to the traditional approach of face-to-face discourse. Given that this is their first experience in this subject discipline, the main shortfalls are the learning curve and technological constraints. Students need to be familiar with the wikis on UM Spectrum. WiLearn may not meet the current practices and expectations due to technological limitations with regard to using the wiki on Moodle. Learning enhancement may be realised if sufficient technological support was offered. Some frustrations may arise during this process, as evident in the following responses:

It is a cumbersome procedure to learn with the wiki on UM Spectrum. Technology should be used for time- and resource-saving, and not be used to create more troubles.(R8)

Wasted a lot of time due to being unfamiliar with the wiki system (R9)

To summarise, the PGW results supported the hypothesis that WiLearn enhancement may be developmentally constructed on wikis; therefore, cognitive skills are mediated by the text/language that is registered on the wikis for developing mental activity, including (1) reflective and deeper review and (2) augmentative communication and collaboration. Yet, the poor "machinery" of the wikis on Moodle may be an obstacle for achieving ZPD. The disruption of the polarity between the technological expectations 
and limitations is obvious. In addition, the research participants from Chinese language studies appear respectful of each other and polite with regard to opposing views without providing strong critical and reflective feedback as required. For the effective use of wikis, the change practice from 'courteous' to critical and reflective feedback is necessary.

\section{Social presence $(S P)$}

Table 2 presents an overview of students' engagement and their activity reports. It appears that there is no direct relationship between the amount of engagement and students' final grades. That is to say that the quantitative analysis of the engagement activity is trivial with regard to the level of socially constructive learning. In contrast with Zokor's (2009) finding, we argue that public visibility of students' engagement in the tasks does not simply reveal enhancement of their own knowledge constructions or help them develop a deeper understanding of the subject matter concepts.

Table 2

Overall view of the participants and their activity reports

\begin{tabular}{ccccc}
\hline Respondents & Group & $\begin{array}{c}\text { Activity Report - Engagement } \\
\text { with Constructing the Wikis }\end{array}$ & $\begin{array}{c}\text { Wiki } \\
\text { Version }\end{array}$ & $\begin{array}{c}\text { Final } \\
\text { Grade }\end{array}$ \\
\hline R1 & 1 & 9 & 24 & B \\
R2 & 3 & 5 & 49 & B+ \\
R3 & 3 & 5 & 49 & B+ \\
R4 & 3 & 15 & 49 & B+ \\
R5 & 4 & 12 & 58 & $\mathrm{~A}-$ \\
R6 & 3 & 9 & 49 & $\mathrm{~B}+$ \\
R7 & 4 & 22 & 58 & $\mathrm{~A}-$ \\
R8 & 2 & 9 & 41 & $\mathrm{~A}$ \\
R9 & 2 & 15 & 41 & $\mathrm{~A}$ \\
R10 & 2 & 8 & 41 & $\mathrm{~A}$ \\
R11 & 1 & 4 & 24 & $\mathrm{~B}$ \\
R12 & 1 & 4 & 24 & $\mathrm{~B}$ \\
R13 & 2 & 8 & 41 & $\mathrm{~A}$ \\
R14 & 4 & 18 & 58 & $\mathrm{~A}-$ \\
R15 & 1 & 5 & 24 & $\mathrm{~B}$ \\
\hline
\end{tabular}

Previous literature acknowledges that the relationship between the degree of "openness" among WiLearn group participants and the individuals' engagement activities facilitates the social construction of knowledge. The present result reveals a more complex relationship between these two factors. Consider the following contrasting experiences of participants, which are categorised by their academic performance:

It is too formal and stereotypical to discuss coursework online through a wiki. I think that real interactions and face-to-face discussions create more opportunities for learning creativity and pleasure (a participant who engaged with WiLearn many times and who had an excellent overall academic performance).

This is a fair and unbiased method for open discussion and peer assessment (a participant who engaged with WiLearn many times and who had an average overall academic performance).

Yes, I would like to use the wiki because I can view different opinions and see more useful, concise and straight forward comments than are available in the intuitive feedback from face-to-face (a participant who engaged with WiLearn a limited number of times and who had a less satisfactory overall academic record).

One common argument that emerged from the students' opinions was that the use of a wiki for peer review was too formal, rigid and less creative than discussions, which some felt were helpful in terms of being more diverse, open and fair. The level of engagement and activity seemed to be irrelevant for having a positive experience. It is interesting to note that students who felt that the wiki was too formal 
and less creative may have been more knowledgeable than those who preferred the wiki. We have no intention of drawing the pedantic conclusion that "good students do not like to use the wiki and average students prefer to use wiki for peer review". We believe that, intuitively, all students have certain intellectual capabilities and learning styles. Given that intellectual ability varies between individuals, instruction needs to be designed to address the differences. Students used to having face-to-face discourse perceive WiLearn as rigid and "operational". However, quieter or less capable students using the wikis for peer review provide justification and feedback with confidence and at their own pace. This finding indicates that this socially inclusive learning process makes up for the lack of affective face-to-face communication. Some students even feel that their online communication with other students is connected and reflected in a constructive manner, as expressed in the following responses:

I could view the comments repeatedly and improve the communication among students.

I can see different opinions and more useful, concise and straight-forward comments than are available in the intuitive feedback from face-to-face.

I can see the comments clearly each time I visit the wiki. I could ponder and reflect repeatedly and edit clearly. We have deeper, connected and repetitive discussions regarding the same agenda. Without the use of the wiki, we would only be able to provide superficial comments and discussion with simpler, perfunctory editing. Yet, there is the possibility that your peers may not adopt or accept your views.

Nevertheless, our findings indicate that social presence should include more than social information exchanges and collaborative editing. The nature of the socially constructive learning process is intertwined with language and "self-spaces" on the wikis, which facilitate and transform mental activity for the ZPD as suggested by Vygotsky (1978). The degree of "openness" and social presence among the WiLearn group participants was insufficient given the educational culture of Chinese language studies in Malaysia. This phenomenon will be further analysed with regard to the OGW.

\section{Outcome of Group Work $(O G W)$}

The assessment of the final outcome included (1) the wiki's content; (2) how the wiki was used for collaborative work and critical discussion; and (3) the students' performances on the peer review. Most of the works were well developed with rich content. An example of a final WiLearn outcome is shown as follows:

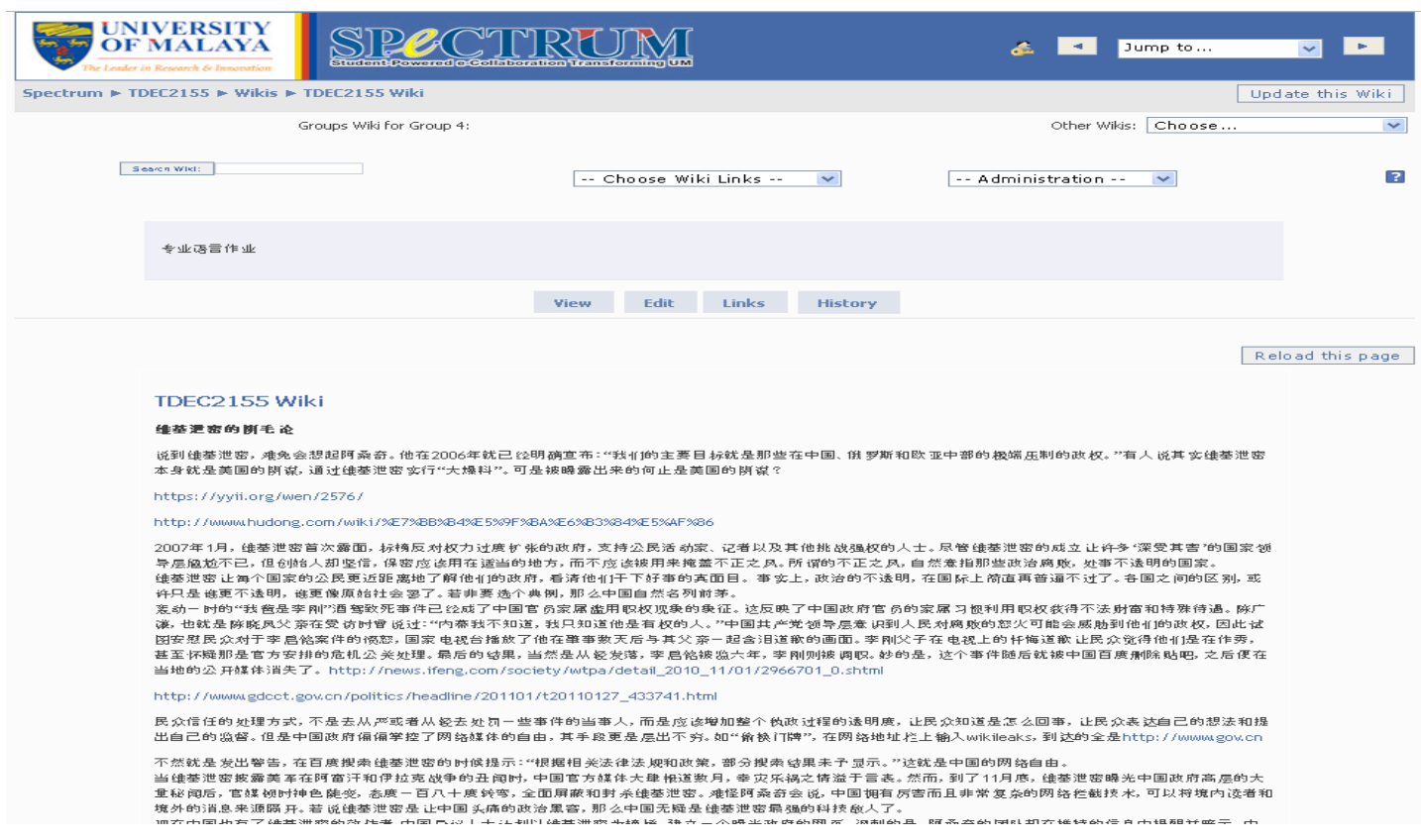

Figure 2. Working interface of a WiLearn outcome 
Instrumentally, the pedagogical design of WiLearn noticeably enhanced the students' learning experiences for Chinese language studies. Generally, students were courteous and intuitive when providing peer review and comments in a face-to-face approach that was based on the teaching experiences of the local agency. The wiki facilitated deeper communication of both linguistic and inclusive agendas. Students who provided reflective discussion and peer review comments during the WiLearn process experienced proximal learning development through the sharing of their experiences and their final grades. Findings suggest that the wiki facilitated an important educational principle for Chinese students, which was Vygotsky's ZPD (1978). Principally, we agree with this claim, yet, there is no evidence that the participants' use of the wikis fundamentally changed their own knowledge constructions or that they developed a deeper understanding of the concepts through transformative dialogue due to the ZDD. Although the participants used the wikis for learning and discourse, the fundamental learning practice remained traditional, for example, the peer review and co-editing practices on WiLearn demonstrated students' submissive educational practice of "listen to what I say" or "do as I say". We suggest that less critical discourse and review among the students was evident, but more critical reflection was found with regard to retrieving others' comments through WiLearn. The present finding is distinct from previous studies, not with regard to the functional use of wikis, but regarding the socialcultural relation. Moreover, students typically explain why an assignment was edited or deleted, which is part of the culture of courtesy justification for mutual understanding. WiLearn does not provide this feature during collaborative authoring and editing. The local agency requested that participants manually develop a summary table to record amendments, such as including the date of editing and a justification for the edit. Figure 3 presents an example:

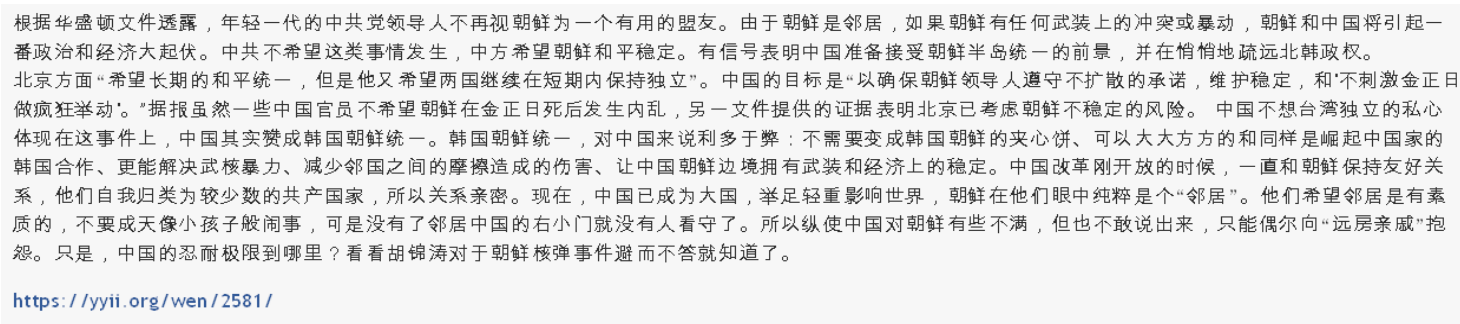

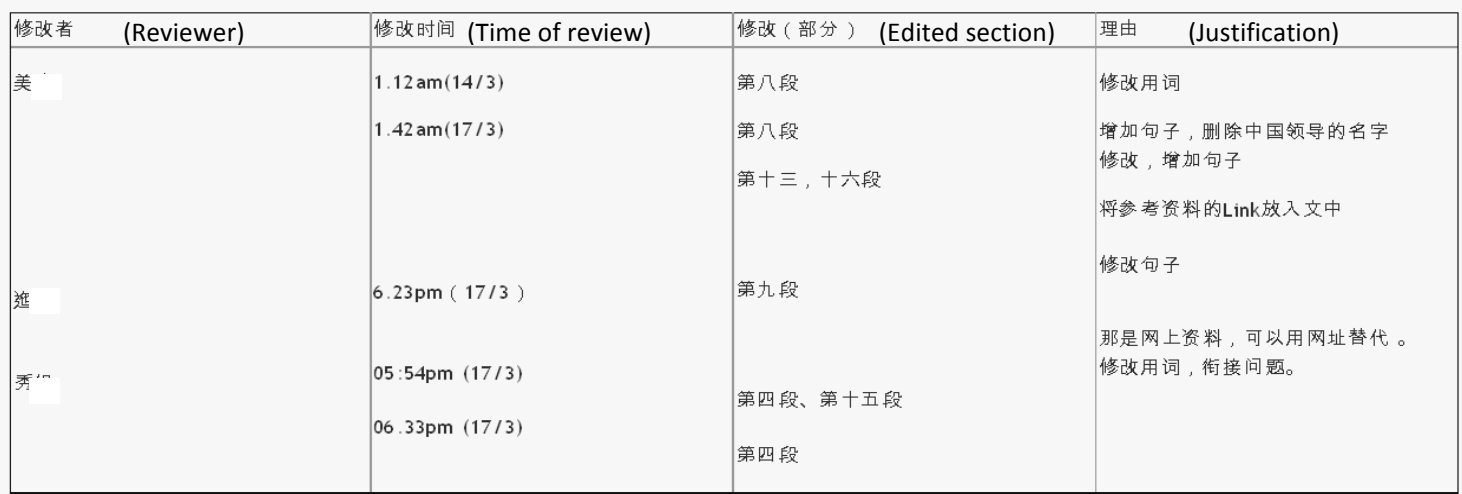

Figure 3. Justification table

\section{Consolidation of the ZPD and/or the ZDD effects on the hypothesis}

Based on the findings from the PGW, SP and OGW, we suggest that an evidence-based value system for WiLearn is necessary. Our argument, at this point, is that it is insignificant if WiLearn adds no value to face-to-face peer assessment, i.e., to the ZDD effects (see Figure 4). WiLearn can only become part of the learning ecosystem for students from Chinese language studies when the ZPD is developed (see Figure 5). Using WiLearn research, we demonstrate disruptive effects in that the ZDD is superior for a number of inference issues but inferior for learning enhancement during constructive experiences. Wikis users' 
learning enhancement may only be constructed during social interactions on wikis that have historical records. The polarity between the ZPD and the ZDD lies not in the functional use of wikis, but in the pedagogical information available for participants from Chinese language studies in Malaysia, especially with regard to changes in the educational practice of critical peer review and discourse that are evident with the use of wikis.

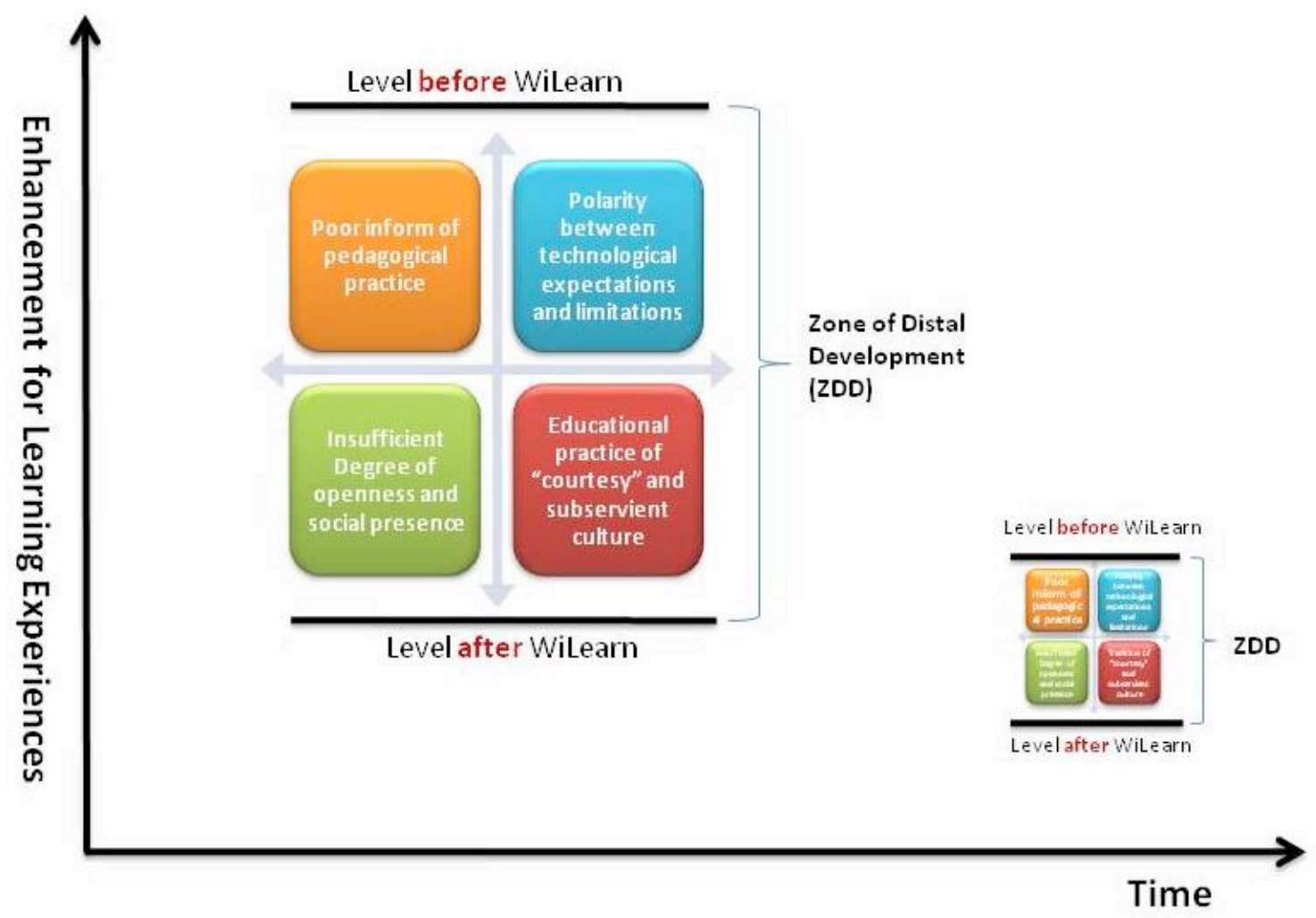

Figure 4. The WiLearn's effects of ZDD

Thus, we assert that, in higher educational contexts, both students and educators have the choice of optimising the effects of the ZPD and minimising the effects of the ZDD. We label this choice as the shared value model (see Figure 6). We agree with Cole's (2009) notion that current literature related to the use of wikis in higher education mainly "seek to promote positive elements of use" (p. 146). The findings report that the zone of distal development for WiLearn is a constructive lesson for other practitioners. We show both the constructive and disruptive aspects of the WiLearn experience in juxtaposition (see Table 3). To present the conditions in which innovative adoption of wikis occurs in Chinese language studies, the shared value model focuses on the practices of the ZPD (i.e., augmentative collaboration and inclusive learning) and the disruption of the ZDD (i.e., the tradition of subservient culture and poor inform of pedagogical practice). Certainly, we do not naively argue that the use of wikis provides students' with better learning experiences and creates the ZPD in Chinese language studies. Our question is that, if wikis increasingly become part of the educational ecosystem, how do current practices become attentive to individual learning enhancement and support students by providing them with a choice regarding the ZPD? The polarity of the ZPD and the ZDD makes students aware of the shared value and forces them to make a choice when engaged in learning and teaching experiences. 


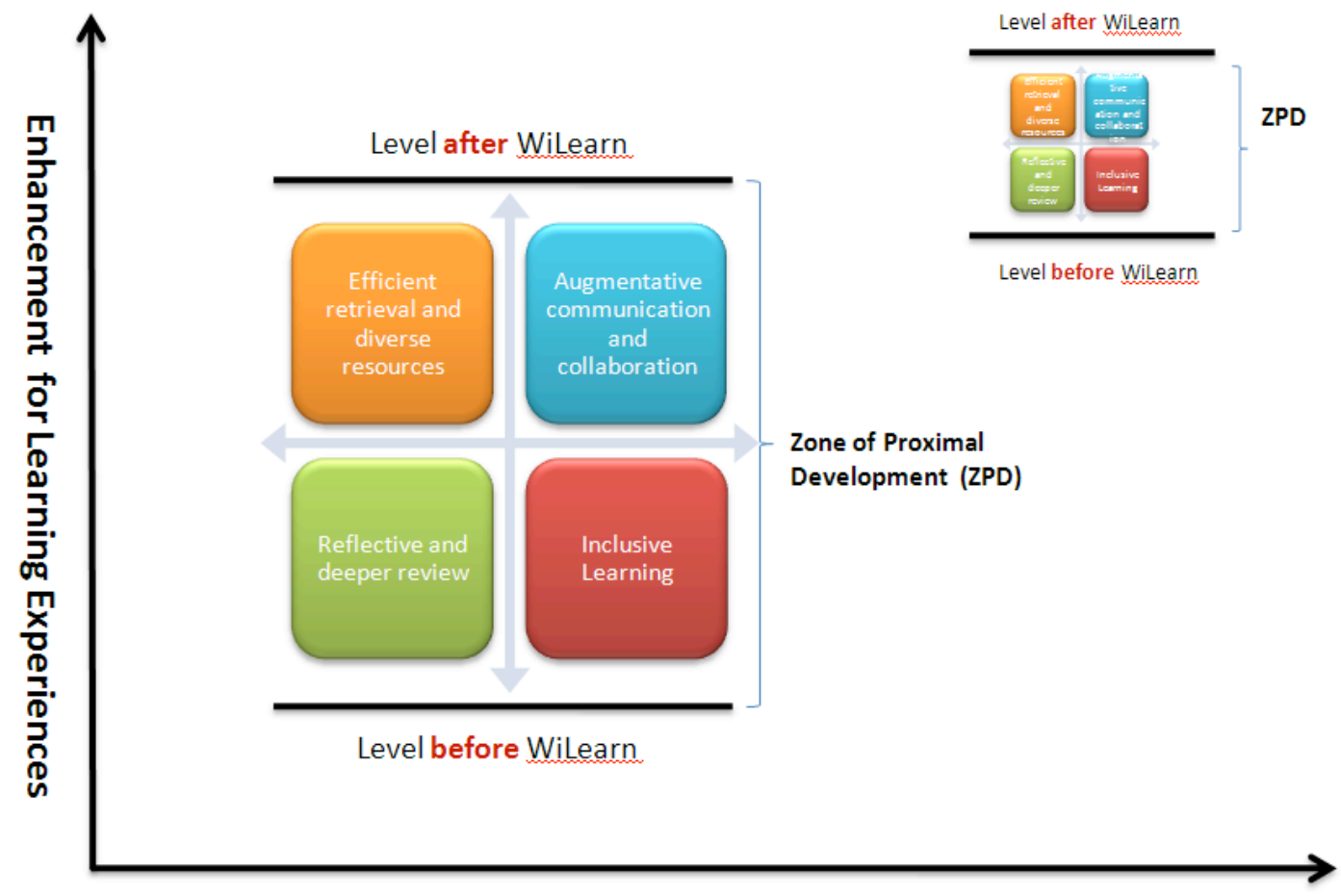

Time

Figure 5. The WiLearn effects on the ZPD

Table 3

The polarity of the ZPD and the ZDD

\begin{tabular}{lll}
\hline \multicolumn{1}{c}{ ZPD } & \multicolumn{1}{c}{ ZDD } \\
\hline $\begin{array}{l}\text { Efficient retrieval and diverse } \\
\text { resources }\end{array}$ & $\bullet \begin{array}{l}\text { Polarity between technological } \\
\text { expectations and limitations }\end{array}$ \\
$\bullet \begin{array}{l}\text { Augmentative communication and } \\
\text { collaboration }\end{array}$ & $\begin{array}{l}\text { Poor inform of pedagogical } \\
\text { practice }\end{array}$ \\
$\bullet \quad$ Reflective and deeper review & $\bullet \begin{array}{l}\text { Educational practice of "courtesy" } \\
\text { and subservient culture }\end{array}$ \\
& $\bullet \quad \begin{array}{l}\text { Insufficient degree of openness } \\
\text { and social presence }\end{array}$ \\
\hline
\end{tabular}

Taking into account the effects of the ZPD and the ZDD on the shared value model, we suggest a few principles of learning with wikis that are based on WiLearn experiences. The following principles may act as guidelines for practitioners who use wikis for learning and teaching:

- Pedagogical preparation for WiLearn implementation is a must. From the perspective of the shared value model, students can be pedagogically prepared and motivated by this process. The ZPD effects with regard to wikis are pertinent to the implementation policy. This includes specific references to how wikis contribute to improving reflective review, inclusive learning and augmentative collaboration. Challenges, such as the polarity between technological expectations and limitations, can be overcome with strong pedagogical information and practices. 
- Recognise the educational practices of courtesy and subservient culture that may lead to less critical peer review and insufficient degrees of openness. Consider a range of good practices for the critical use of wikis for peer review and provide related information to support a fair boundary in terms of students who are neither advantaged nor disadvantaged by the social presence.

- Pre-technical training and post-training support should be offered to students in Chinese language studies to decrease the polarity between technological expectations and limitations.

- An additional column should be inserted on the wikis by default that allows the option of writing justifications for the changes provided by peers.

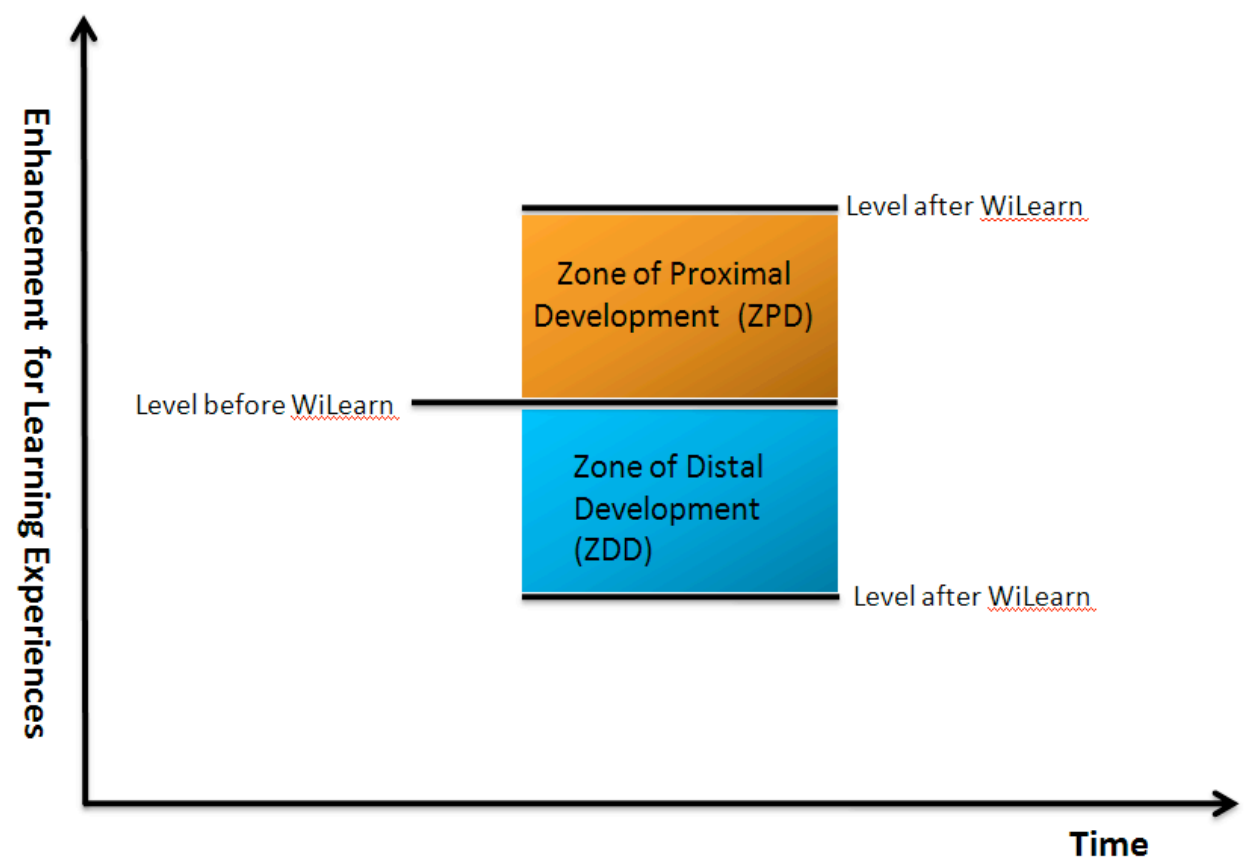

Figure 6. Shared value model for WiLearn

\section{Limitations and future work}

This study explored the WiLearn experience with the goal of profiling the ZPD and the ZDD. However, the qualitative method employed in this research may not achieve the validity standards needed to generalise the findings, as required by a quantitative content analysis. This view is expressed in a different way by Gill (1995) and Rist (1997), who defend qualitative findings as leading to considerable theoretical developments that can be generalised internationally. We argue that, although the sample size for this WiLearn study was small, we analysed the evidence in a systematic manner and provided a theoretical development that should be examined with larger sample sizes and in different contexts, such as in other language studies, to investigate the diversity of possibilities for the ZPD and the ZDD. The tension and polarity between constructive and disruptive uses of wikis should be investigated in the future across disciplines in higher education.

\section{Conclusions}

WiLearn is a collaborative research project with the UK and Malaysia that examines the use of wikis during peer review practices with students in Chinese language studies in Malaysia, in accordance with the UK's TEL experience. This study investigated the effects of wiki use on the ZPD and/or the ZDD of students in Chinese language studies and the polarity of the ZPD and the ZDD. The results support current literature indicating that wikis provide community learning and contribute to the process of collective learning and collaboration. Guth (2007) suggested that students who contribute to wikis feel more responsible for their coursework and develop their skill of critical criticism. Principally, we agree with this claim. However, we assert that less critical discourse was evident among our participants, but 
more critical reflection was evident with regard to retrieving peers' comments through WiLearn. The polarity between the ZPD and the ZDD lies not in the functional use of wikis but in the degree of openness and social presence of the participants in Chinese language studies, especially with regard to educational practices and pedagogical changes in the critical peer review process and the discourse regarding the use of wikis. Thus, fewer cognitive skills are mediated by the Chinese text/language that is registered on wikis for developing mental activity given the insufficient social-cultural relations. Moreover, whenever possible, a shared value system can be offered to students to amplify the learning enhancements and pedagogical change. These principles can possibly transform challenges into opportunities for the learning cultural ecosystem of Chinese language studies.

\section{Acknowledgements}

We sincerely appreciate the research funding from the University of Malaya (Project No: RG365-12HNE).

\section{References}

Abram, D. (1996). The spell of the sensuous. New York: Vintage.

ADeC (2011). Academic Development Centre. Retrieved July 28, 2011 from http://adec.um.edu.my/mission.html

Ajjan, H. \& Hartshorne, R. (2008). Investigating faculty decisions to adopt web 2.0 technologies: Theory and empirical tests. Internet and Higher Education, 11, 71-80.

Anthony, D., Smith, S., \& Williamson, T. (2005). Explaining quality in internet collective goods: Zealots and good Samaritans in the case of Wikipedia, the MIT Fall 2005 Innovation and Entrepreneurship Seminar. Retrieved July 28, 2011 from http://web.mit.edu/iandeseminar/Papers/Fall2005/anthony-pdf

Bold, M. (2006). Use of wikis in graduate course work. Journal of Interactive Learning Research, 17(1), 5-14.

Cohen, L., Manion, L., \& Morrison, K. (2001). Research methods in education (5 ${ }^{\text {th }}$ ed.). London: Routledge Falmer.

Cole, M. (2009). Using Wiki technology to support student engagement: Lessons from the trenches, Computers \& Education 52 (1) 141-146.

Conole, G., Laat, M., D., Dillon, T., \& Darby, J. (2008). Disruptive technologies, pedagogical innovation: what's new? Findings from an in-depth study of students' use and perception of technology. Computers \& Education, 50(2), 511-524.

Conole, G. (2010). Facilitating new forms of discourse for learning and teaching: harnessing the power of Web 2.0 practices. Open Learning, 25(2), 141-151.

Connolly, T. M., Stansfield, M., \& Hainey, T. (2011). An alternate reality game for language learning: ARGuing for multilingual motivation, Computers \& Education, 57(1) 1389-1415.

Cortazzi, M., \& Hall, B. (1999). Vygotsky and learning. Education Libraries Journal, 42(3), 17-21.

Deborah J. L., \& Bodrova, E. (2001). Lev Vygotsky: Playing to learn. Scholastic Early Childhood Today, $15(4), 48$.

Denscombe, M. (2002). The good research guide: For small-scale social research projects. Buckingham: Open University Press.

Engstrom, M., \& Jewett, D. (2005). Collaborative learning the wiki way. TechTrends, 49(6), 12-16.

Flick, U. (1998). An introduction to qualitative research. London: Sage Publications. 
Gill, J. (1995). Building theory from case studies. In B. Richardson, L. Montanheiro, \& B. O. Cinneide (Eds.), How to research, write, teach and publish management case studies (pp. 17-27). Sheffield: PAVIC.

Guth, S. (2007). Wikis in education: is public better? Conference Proceedings of the 2007 International Symposium on Wikis, WikiSym'07, Montreal, pp. 61-68. Retrieved April 17, 2012 from http://www.wikisym.org/ws2007/_publish/Guth_WikiSym2007_IsPublicBetter.pdf

Gwella. (2010). Enhancing learning \& teaching through technology for higher education in Wales. Retrieved April 19, 2012 from http://www.heacademy.ac.uk/resources/detail/nations/wales/gwella

Haley, E. R., Collins, G. B., \& Coe, D. J. (2008). The wonderful world of wiki benefits students and instructors. IEEE Potentials, 27(2), 21-26.

Hull, D. M., \& Saxon, T. F. (2009). Negotiation of meaning and co-construction of knowledge: An experimental analysis of asynchronous online instruction. Computers \& Education, 52(3), 624-639.

Hwang, J. M., \& Brummans, B. (2011). Learning about media effects by building a wiki community: students' experiences and satisfaction, In C. Wankel (Ed.), Teaching arts and science with the new social media(pp. 39-59). Bingley, UK: Emerald.

Jaramillo, J. A. (1996). Vygotsky's sociocultural theory and contributions to the development of constructivist curricula. Education, 117(1), 133-141.

JISC. (2011). Joint Information Systems Committee. Retrieved July 24, 2011 from http://www.jisc.ac.uk/

Jones, N., Blackey, H., Fitzgibbon, K., \& Chew, E. (2010). Get out of MySpace! Computers \& Education, 54(3), 776-782.

Joubert, M., \& Wishart, J. (2012). Participatory practices: Lessons learnt from two initiatives using online digital technologies to build knowledge, Computers \& Education, 59(1), 110-119.

Kim, D. (2011). Incorporating podcasting and blogging into a core task for ESOL teacher Candidates, Computers \& Education, 56(3) 632-641.

Lai, Y. C., \& M. W. Ng, (2011). Using wikis to develop student teachers' learning, teaching, and assessment capabilities, Internet and Higher Education, 14(1), 15-26.

Lamb, A., \& Johnson, L. (2007). An information skills workout: wikis and collaborative writing. Teacher Librarian, 34(5), 57-59.

Laughton, P. (2011). The use of wikis as alternatives to learning content management systems. Journal of the Electronic Library, 29(2), 225-235.

Laurillard, D. (2002). Rethinking university teaching (2nd ed.). London: Routledge Falmer.

Leading, D. (2010). Teaching tips: using wiki to collaborate on a study guide. Journal of Information Systems Education, 21(1), 5-13.

Leong, D. J., \& Bodrova, E. (2007). Vygotsky's Zone of Proximal Development. NAECS/SDE: Of Primary Interest, 2(4). Retrieved July 21, 2008 from http://naecs.crc.uiuc.edu/newsletter/volume2/number4.html (viewed 21 July 2008)

Mak, B., \& Coniam, D. (2008). Using wikis to enhance and develop writing skills among secondary school students in Hong Kong. System, 36(3), 437-455.

McConnell, D. (2006). E-learning groups and communities. Berkshire: Open University Press. 
Mohamed, A. E. (2011). e-Learning in Malaysian Higher Education Institutions: Status, Trends and Challenges. Putrajaya: Ministry of Higher Education. Retrieved August 3, 2011 from http://www.scribd.com/doc/53498507/E-learning-in-Malaysian-Higher-Education-Institutions

Murugesan, S. (2007). Understanding Web 2.0. IT Professional, 9(4), 34-41.

Ng, E. M. W., \& Lim, C. P. (2011). Editorial for special issue: The Internet and teacher education - An Asian experience. Internet and Higher Education, 14(1) 1-2.

Nussbaum, M., Alvarez, C., McFarlane, A., Gomez, F., Claro, S., \& Radovic, D. (2009). Technology as small group face-to-face Collaborative Scaffolding. Computers \& Education, 52(1), 147-153.

Pear, J. J., \& Crone-Todd, D. E. (2002). A social constructivist approach to computer-mediated instruction, Computers \& Education, 38(1-3), 221-231.

Richardson, W. (2006). Blogs, wikis, podcasts, and other powerful tools for the classrooms. California: Corwin Press, Thousand Oaks.

Rist, R. (1977). On the relations among education research paradigms: from disdain to détente. Anthropology and Education, 8(2), 42-50.

Robertson, I. (2008). Learners' attitudes to wiki technology in problem based, blended learning for vocational teacher education. Australasian Journal of Educational Technology, 24(4), 425-441.

Salmon, G. (2002). E-tivities: The key to active online learning. London: Kogan Page.

Sharpe, R., Benfield, G., Roberts, G., \& Francis, R. (2006). The undergraduate experience of blended elearning: A review of UK literature and practice. UK: The Higher Education Academy.

Shiha, S., Tsenga, S., \& Yangc, C. T. (2008). Wiki-based rapid prototyping for teaching-material design in e-learning grids. Computers \& Education, 51(3), 1037-1057.

Soylu, F. (2009). Academics' views on and uses of wikipedia. Journal of Communication, Culture and Technology, 9(2).

Subramaniam, K. (2007). Teachers' mindsets and the integration of computer technology. British Journal of Educational Technology, 38(6), 1056-1071.

Taylor, S. J., \& Bogdan, R. (1998). Introduction to qualitative research methods: A guidebook and resource. New York: John Wiley \& Son.

Torbert, W. (2001). The practice of action inquiry. In Reason, P. \& Bradbury (Ed), Handbook of Action Research - Participative Inquiry and Practice. London: Sage.

Vaus, D. A. (2001). Research Design in Social Research. London: Sage Publication.

Vygotsky, L.S. (1978). Mind in society: The development of higher psychological processes. Cambridge, MA: Harvard University Press.

Wagner, C. (2004). Wiki: a technology for conversational knowledge management and group collaboration. Communications of the Association for Information Systems, 13(1), 265-289.

Waters, N. L. (2007). Why you cite wikipedia in my class?” Communication of the ACM, 50(9), 15-17. Retrieved July 30, 2011 from http://delivery.acm.org/10.1145/1290000/1284635/p15waters.pdf? ip $=193.63 .146 .211 \&$ CFID $=34879606 \& C F T O K E N=53220737 \&$ acm_ $=1311942541 \_$e 4ff61157f4cf45c43210845971e5814

West, J. A., \& West, M. L. (2009). Using wikis for online collaboration: The power of the read-write web. San Francisco, CA: Wiley \& Sons. 
Weaver, B. D., Keer, H.V., Schellens, T., \& Valcke, M. (2011). Assessing collaboration in a wiki: The reliability of university students' peer assessment. Internet and Higher Education, 14(4), 201-206.

Wilkinson, D. M., \& Huberman, B. A. (2007). Assessing the value of cooperation in Wikipedia. First Monday, 12(4). Retrieved August 3, 2011 from www.firstmonday.org/issues/issue12_4/wilkinson/index.html

Xiao, Y., \& Lucking, R. (2008). The impact of two types of peer assessment on students' performance and satisfaction within a Wiki environment. Internet and Higher Education, 11(3-4), 186-193

Zokor, V. (2009). Factors affecting the way students collaborate in a wiki for English language learning, Australasian Journal of Educational Technology, 25(5), 645-665.

Corresponding author: Chew Esyin, chew.esyin@monash.edu

Australasian Journal of Educational Technology (C) 2014.

Please cite as: Chew, E., \& Ding, S. L. (2014). The Zones of Proximal and Distal Development in Chinese Language studies with the use of wikis. Australasian Journal of Educational Technology, 30(2), 184-201. 